\title{
A note on compact and compact circular edge-colorings of graphs $\left.\right|^{\dagger}$
}

\section{Dariusz Dereniowski and Adam Nadolski}

Department of Algorithms and System Modeling, Gdańsk University of Technology,

ul. G. Narutowicza 11/12, 80-952 Gdańsk

\{nadolski, deren\}eeti.pg.gda.pl

received July 2, 2006, revised July 3, 2008, accepted December 2, 2008.

\begin{abstract}
We study two variants of edge-coloring of edge-weighted graphs, namely compact edge-coloring and circular compact edge-coloring. First, we discuss relations between these two coloring models. We prove that every outerplanar bipartite graph admits a compact edge-coloring and that the decision problem of the existence of compact circular edge-coloring is NP-complete in general. Then we provide a polynomial time 1.5-approximation algorithm and pseudo-polynomial exact algorithm for compact circular coloring of odd cycles and prove that it is NP-hard to optimally color these graphs. Finally, we prove that if a path $P_{2}$ is joined by an edge to an odd cycle then the problem of the existence of a compact circular coloring becomes NP-complete.
\end{abstract}

Keywords: edge-weighted graph, compact edge-coloring, interval edge-coloring, compact circular edge-coloring, NP-completeness, approximation algorithm.

\section{Introduction}

In the paper we consider two variants of edge-coloring of weighted graphs. Let $(G, w)$ be a weighted graph, where $G=(V(G), E(G))$ and $w: E(G) \mapsto \mathbb{N}$. For a given positive real $r$ denote by $I^{r}$ the interval $[0, r]$ and by $C^{r}$ a circle of circumference $r$. Moreover, let $\mathcal{J}\left(I^{r}\right)$ and $\mathcal{A}\left(C^{r}\right)$ denote the set of all open subintervals of $I^{r}$ and the set of all open arcs of $C^{r}$, respectively. Then, an edge r-coloring of $G$ is an assignment $c: E(G) \mapsto \mathcal{J}\left(I^{r}\right)$ such that $|c(e)|=w(e)$ for every edge $e$ and $c\left(e_{1}\right) \cap c\left(e_{2}\right)=\emptyset$ for every pair of adjacent edges $e_{1}, e_{2}$. For a vertex $v \in V(G)$ let $B_{v}$ denote the set of the edges that are incident to $v$. An edge $r$-coloring is said to be compact at $v$, if $\overline{\bigcup_{e \in B_{v}} c(e)}$, namely the closure of the union of the intervals assigned to the edges incident to $v$, forms an interval. An edge $r$-coloring that is compact at every vertex $v \in V(G)$ is said to be compact.

Notice that a circle $C^{r}$ can be considered as an interval $I^{r}$ with identified endpoints. In this way $\mathcal{J}\left(I^{r}\right)$ is transformed into a subset of $\mathcal{A}\left(C^{r}\right)$. Therefore, these arcs can be denoted as $(a, b)$, where $a<b$. The arcs of $\mathcal{A}\left(C^{r}\right)$ that are not inherited from $\mathcal{J}\left(I^{r}\right)$ are the open arcs containing point 0 , therefore they can be

\footnotetext{
${ }^{\dagger}$ Both authors are supported in parts by Foundation for Polish Science and Ministry of Science grant N516 029 31/2941. 


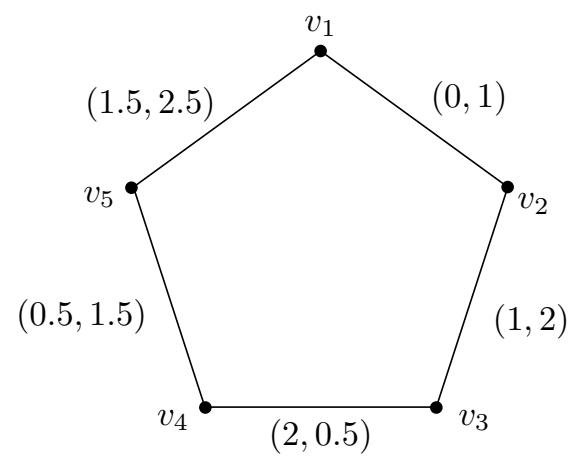

Fig. 1: An optimal circular edge-coloring of $C_{5}$.

denoted as $(a, b)$, where $b<a$. These notions motivate us to transform the definition of edge-coloring in such a way that arcs of a circle, instead of intervals, are assigned to the edges. A circular edge r-coloring of $(G, w)$ is a mapping $c: E(G) \mapsto \mathcal{A}\left(C^{r}\right)$ such that $|c(e)|=w(e)$ for every edge $e$ and $c\left(e_{1}\right) \cap c\left(e_{2}\right)=\emptyset$ for every pair of adjacent edges $e_{1}, e_{2}$. In the same way as above we provide the definition of compactness for this coloring model. Namely, a circular edge $r$-coloring is said to be compact at vertex $v \in V(G)$ if $\overline{\bigcup_{e \in B_{v}} c(e)}$ forms an arc of $C^{r}$ or is equal to $C^{r}$. Finally, a circular edge $r$-coloring is compact if it is compact at every vertex $v \in V(G)$.

In the paper we consider edge-colorings only, therefore we often write circular $r$-coloring and $r$ coloring instead of circular edge $r$-coloring and edge $r$-coloring, respectively. Moreover, in the case $r$-coloring could be misled with circular $r$-coloring, we write standard $r$-coloring instead of $r$-coloring.

At this point we would like to notice that the restriction of the edge weights to positive integers is for convenience merely. Suppose the weights were rational and $c$ was a standard or circular $r$-coloring. Then, we could multiply all the weights by $M$, the least common multiple of the denominators of the weights, making all of the weights positive integers. If we also multiplied by $M$ the endpoints of all the colors assigned by $c$, we would obtain a standard or circular $(M \cdot r)$-coloring. Moreover, this transformation would preserve the compactness property in both coloring models.

In both cases our objective is to minimize the parameter $r$, namely the length of an interval or the length of a circle. In the following, we name the parameter $r$ as the number of colors. Clearly, every $r$-coloring can be transformed into a circular $r$-coloring. However, the opposite transformation is not always possible. Fig. 1 depicts an optimal circular edge-coloring of $C_{5}$ with all weights equal to 1 [5]. This coloring uses 2.5 colors, while a standard coloring would require at least 3 colors. Notice that the transformation of an $r$-coloring into a circular $r$-coloring preserves the property of compactness.

Both coloring variants can be applied in scheduling theory to represent schedules with no-idles in models such as biprocessor tasks system or open shop. Consider an automated production system in which there is a set of tasks, each requiring the simultaneous use of two preassigned machines (processors, tools, etc.). This situation can be modeled by a graph, whose vertices correspond to the machines and the edges correspond to the tasks. Then, each schedule of makespan $r$ can be represented by an $r$-coloring of such a graph. Moreover, if the production process proceeds in a periodic way, i.e. whenever one set of tasks is completed, the following one is started immediately, the schedule is modeled by a circular edge-coloring. In some industrial applications, like chemistry or metallurgy, the processors have to work 
without idle times due to technological or economical constraints. It occurs that a schedule with no idles on all processors corresponds to a compact coloring in both standard and periodic setting. This motivates our considerations of compact coloring models. The reader interested further in relations between compact colorings and schedules fulfilling the no-idle constraint is referred to [3, 8, 9, 12]

There are numerous papers on the problem of compact edge-coloring, often referred to as interval or consecutive edge-coloring. Surveys on this topic can be found in several books that deal with various problems in graph coloring [1, 4, 6]. It is known that not every graph admits a compact coloring even if all weights are equal to 1 and the problem of determining whether a given graph can be colored in a compact manner is NP-hard, even for bipartite graphs [10]. In the case of arbitrary weights the same problem is NP-hard even for odd cycles [3]. On the other hand, paths and even cycles admit compact edge-colorings. However, for these graphs an optimal compact coloring cannot be determined in polynomial time, unless $\mathrm{P}=\mathrm{NP}[2]$.

The compact circular coloring variant has attained less attention in the literature. The paper [7] studies properties of this coloring model in the case of unit weights. The author constructed graphs, for which optimal compact circular colorings are determined and graphs, for which it is proved that there are no compact circular colorings. In the papers [11, 12] the authors consider compact circular coloring of bipartite graphs in the context of application of this coloring model in scheduling, in the cyclic version of open shop.

Clearly, no compact or compact circular $r$-coloring exists for $r<\Delta(G, w)$, where $\Delta(G, w)=$ $\max _{v \in V(G)}\left\{\sum_{e \in B_{v}} w(e)\right\}$ denotes the weighted degree of $(G, w)$. Below, we cite an interesting result used in the following sections, which classifies graphs for which there exists a compact circular $r$-coloring for every weight function $w$ and every $r \geq \Delta(G, w)$.

Theorem 1 ([11]) For every graph $G$ the following two statements are equivalent:

(i) for every weight function $w$ and every $r \geq \Delta(G, w)$ there exists a compact circular $r$-coloring of $(G, w)$;

(ii) $G$ is bipartite and outerplanar.

This paper is organized as follows. In the next section (Sec.2) we discuss the relations between compact and circular compact colorings. These considerations yield a characterization of the graphs which admit a standard compact coloring with arbitrary weights on the edges. Moreover, we prove that the decision problem of existence of a circular compact coloring is NP-complete. Then, we study the problem of compact circular coloring of odd cycles in Section 3. We prove that a compact circular coloring exists for every weighted odd cycle and provide an approximation algorithm. Moreover, we prove that an optimal coloring cannot be determined in polynomial time, unless $\mathrm{P}=\mathrm{NP}$ and construct a pseudo-polynomial exact algorithm. Finally, we show that joining a path $P_{2}$ to a vertex of a cycle makes the problem of colorability NP-hard.

\section{Relations between Standard and Circular Compact Colorings}

As it was noticed in the introduction, a compact $r$-coloring can be transformed into compact circular $r$ coloring. The following proposition strengthens this statement. Its proof is based on the ideas presented in [7] for non-weighted colorings. 
Proposition 2 Suppose that a graph $G$ with a weight function $w$ admits a compact standard edge $r$ coloring for some $r \geq \Delta(G, w)$. Then there exists a compact circular s-coloring of $(G, w)$ for every $s \geq \Delta(G, w)$.

Proof: Let $c$ be a compact $r$-coloring and let $s \geq \Delta(G, w)$. For a given edge $e$ let $c_{1}(e)$ and $c_{2}(e)$ denote the left and right endpoint of the interval $c(e)$, respectively. Moreover, for $x \geq 0$ we denote by $[x]_{s}$ the unique $y \in[0, s)$, such that $x=y+i \cdot s$ for some integer $i$. We construct an assignment $\bar{c}: E(G) \mapsto \mathcal{A}\left(C^{s}\right)$ given by the following formula

$$
\bar{c}(e)=\left(\left[c_{1}(e)\right]_{s},\left[c_{2}(e)\right]_{s}\right) .
$$

We claim that $\bar{c}$ is a compact circular $s$-coloring.

Clearly, $|c(e)|=w(e)$ for every edge $e$. Let $e_{1}$ and $e_{2}$ be two adjacent edges. Without loss of generality suppose that $c_{1}\left(e_{1}\right)<c_{2}\left(e_{1}\right) \leq c_{1}\left(e_{2}\right)<c_{2}\left(e_{2}\right)$. Then, $c_{2}\left(e_{2}\right) \leq c_{1}\left(e_{1}\right)+\Delta(G, w)$, since $c$ is compact. This together with $s \geq \Delta(G, w)$ yields $\bar{c}\left(e_{1}\right) \cap \bar{c}\left(e_{2}\right)=\emptyset$, which proves that $\bar{c}$ is a circular $s$-coloring. The proof that $\bar{c}$ is compact is straightforward.

Suppose that for a given weighted graph $(G, w)$ we have a compact circular $r$-coloring for some $r>$ $\sum_{e \in E(G)} w(e)$. Then, there exists a point $x \in C^{r}$ such that $x \notin c(e)$ for any edge $e$. Without loss of generality we may assume that $x=0$. Therefore, every arc $c(e)$ can be transformed to an appropriate interval from $\mathcal{J}\left(I^{r}\right)$. In this way we construct an $r$-coloring of $(G, w)$, which is compact. This gives us the following proposition.

Proposition 3 If for a given graph $G$ and a weight function $w$ there exists a compact circular $r$-coloring, where $r>\sum_{e \in E(G)} w(e)$, then there exists a compact r-coloring of $(G, w)$.

Propositions 2 and 3 together with Theorem 1 yield the following theorem.

Theorem 4 For every graph $G$ the following two conditions are equivalent:

(i) for every weight function $w$ there exists a compact $r$-coloring of $(G, w)$ for some $r \geq \Delta(G, w)$;

(ii) $G$ is bipartite and outerplanar.

Proof: $(i) \Rightarrow(i i)$ : Let $w$ be a given weight function. From $(i)$ we obtain that $(G, w)$ has a compact $r$-coloring for some $r \geq \Delta(G, w)$, which together with Proposition2 yields that $(G, w)$ admits a compact circular $s$-coloring for every $s \geq \Delta(G, w)$. Since $w$ is arbitrary, Theorem 1 implies that $G$ is both bipartite and outerplanar.

$($ ii $) \Rightarrow(i)$ : Let $w$ be any weight function. Put $r=\sum_{e \in E(G)} w(e)+1$. From Theorem 1 it follows that there exists a compact circular $r$-coloring. Proposition 3 implies that there exists an $r$-coloring of $(G, w)$ that is compact.

Note that the proof of Theorem 1 from [11] together with the proof of Proposition 3 leads to a polynomialtime algorithm for compact coloring of weighted outerplanar bipartite graphs. On the other hand, the problem of determining the optimal compact coloring for these graphs is NP-hard, since it is already NP-hard for paths and even cycles [2].

In the following proposition we prove that one cannot determine in polynomial time whether a given weighted graph admits any compact circular $r$-coloring, unless $\mathrm{P}=\mathrm{NP}$. 
Proposition 5 The following problem is NP-complete: "Given a weighted graph $(G, w)$. Does there exist a real $r$ such that $(G, w)$ has a compact circular $r$-coloring?”.

Proof: First we prove that the problem belongs to NP. Note that it is not straightforward, since $r$ and colors are from continuous domain. It suffices to argue that the answer is positive if and only if there exists a rational $\bar{r}=p / q, p, q \in \mathbb{N}, \operatorname{gcd}(p, q)=1, q \leq p \leq \sum_{e \in E(G)} w(e)$ and a compact circular $\bar{r}$-coloring $\bar{c}$ such that $\bar{c}(e)=(\phi(e) / q,(\phi(e)+q \cdot w(e)) / q)$ for every edge $e$, where $\phi(e) \in\{0, \ldots, p-1\}$ and addition is taken modulo $q$.

For convenience we assume that $G$ is connected. Let $c$ be a compact circular $r$-coloring. If $r$ is irrational we claim that there exists a standard compact coloring which leads to the existence of circular compact $\Delta(G, w)$-coloring by Proposition 2. We can assume that there is an edge $e_{0}$ for which $c\left(e_{0}\right)=(0, w(e))$, shifting the colors if needed. Denote by $\ell(e)$ the left endpoint of $c(e)$ for each edge $e$. We have $\ell\left(e_{0}\right)=0$, which together with connectivity of $G$ and compactness of $c$ implies that for each edge $e$ there exists an integer $k_{e}$ such that $\ell(e) \equiv k_{e}(\bmod r)$. Since $r$ is irrational, $k_{e}$ is unique. It is easy to observe that assigning $\left(k_{e}, k_{e}+w(e)\right)$ to each edge $e$ gives a standard compact coloring.

Suppose $c$ is a compact circular $r$-coloring, where $r=p / q$ is rational, $\operatorname{gcd}(p, q)=1$. We can assume 0 is the left endpoint of a color of some edge, shifting the colors if needed. Then, the compactness property implies that $c$ satisfies the condition $c(e)=(\phi(e) / q,(\phi(e)+q \cdot w(e)) / q)$ for each edge.

Now we argue that if $p>\sum_{e \in E(G)} w(e)$ then one could construct a compact circular $\Delta(G, w)$ coloring. Suppose that $c$ is a compact circular $(p / q)$-coloring, where $p>\sum_{e \in E(G)} w(e)$. We assume that $c(e)=(\phi(e) / q,(\phi(e)+q w(e)) / q)$ for each edge $e$, where $\phi(e) \in\{0, \ldots, p-1\}$. Then, there exists some $x \in\{0, \ldots, p-1\}$ that $\phi(e) \neq x$ for any edge $e$. Without loss of generality we assume that $x=0$. Since $p$ and $q$ are coprime, for each $y \in\{1, \ldots, p-1\}$ there exists a unique $z(y) \in\{1, \ldots, p-1\}$ such that $q \cdot z(y) \equiv y(\bmod p)$. Using the same argument as that in the proof of Theorem 5 in [7], one can observe that assigning $(z(\phi(e)), z(\phi(e))+w(e))$ to each edge $e$ yields a compact standard edge-coloring. Thus, we can construct a compact circular $\Delta(G, w)$-coloring by Proposition 2

To complete the proof we construct a reduction from a problem which is NP-complete to the considered one. Consider a weighted graph $(G, w)$. Let $S=\sum_{e \in E(G)} w(e)$. We construct a weighted graph $(\bar{G}, \bar{w})$ by adding to $(G, w)$ two new vertices connected by an edge of weight $S+1$. We claim that $(G, w)$ admits a compact standard $r$-coloring for some $r$ if and only if $(\bar{G}, \bar{w})$ admits a circular compact $q$-coloring for some $q$.

Suppose there exists a compact standard $r$-coloring of $(G, w)$. Then, by Proposition 2 there exists a compact circular $q$-coloring of $(\bar{G}, \bar{w})$, where $q=\max \{r, S+1\} \geq \Delta(\bar{G}, \bar{w})$.

Now let $c$ be a compact circular $q$-coloring of $(\bar{G}, \bar{w})$. Such a coloring restricted to $(G, w)$ would be a compact circular $q$-coloring of $(G, w)$. Moreover, $q \geq S+1$, which together with Proposition 3 yields that $(G, w)$ admits a compact standard $q$-coloring.

Therefore, we have reduced the problem of the existence of a compact $r$-coloring for some $r$ to the problem of the existence of a compact circular $q$-coloring for some $q$. Since the former is NP-complete [10], this completes the proof.

\section{Compact Circular Coloring of Odd Cycles}

The results of the previous section imply that no efficient algorithm can construct a compact circular coloring of an arbitrary graph, while the results of [12] provide an algorithm that constructs an optimal 
compact circular coloring of any outerplanar bipartite graph in polynomial time. In the first part of this section we provide an algorithm for compact circular coloring of odd cycles. Note that determining whether there exists a standard compact coloring of an odd cycle is NP-hard [3].

Theorem 6 For every weight function $w: E\left(C_{2 k+1}\right) \mapsto \mathbb{N}$ of an odd cycle there exists a compact circular $r$-coloring for some $r \leq 1.5 \cdot \Delta\left(C_{2 k+1}, w\right)$.

Proof: Denote the consecutive vertices of the cycle as $v_{0}, \ldots, v_{2 k}$ and assume that $w\left(\left\{v_{0}, v_{1}\right\}\right)+$ $w\left(\left\{v_{1}, v_{2}\right\}\right)=\Delta(G, w)$ and $w\left(\left\{v_{0}, v_{1}\right\}\right) \geq w\left(\left\{v_{1}, v_{2}\right\}\right)$. Let

$$
r=w\left(\left\{v_{2 k}, v_{0}\right\}\right)+w\left(\left\{v_{0}, v_{1}\right\}\right)+w\left(\left\{v_{1}, v_{2}\right\}\right) .
$$

The following inequalities hold

$$
w\left(\left\{v_{2 k}, v_{0}\right\}\right) \leq w\left(\left\{v_{1}, v_{2}\right\}\right) \leq 0.5 \cdot \Delta\left(C_{2 k+1}, w\right),
$$

which implies that $r \leq 1.5 \cdot \Delta(G, w)$. We construct an assignment $c: E\left(C_{2 k+1}\right) \mapsto \mathcal{A}\left(C^{r}\right)$ as follows

$$
\begin{aligned}
c\left(\left\{v_{0}, v_{1}\right\}\right) & =\left(w\left(\left\{v_{1}, v_{2}\right\}\right), r-w\left(\left\{v_{2 k}, v_{0}\right\}\right)\right), \\
c\left(\left\{v_{i}, v_{i+1}\right\}\right) & =\left(0, w\left(\left\{v_{i}, v_{i+1}\right\}\right)\right) \quad \text { for } i=1,3, \ldots, 2 k-1, \\
c\left(\left\{v_{i}, v_{i+1}\right\}\right) & =\left(r-w\left(\left\{v_{2 k}, v_{0}\right\}, r\right) \quad \text { for } i=2,4, \ldots, 2 k,\right.
\end{aligned}
$$

where $v_{2 k+1}=v_{0}$. We claim that $c$ is a compact circular $r$-coloring.

First, consider a fixed vertex $v_{i}$, where $i \in\{2,3, \ldots, 2 k\}$. Observe that the colors assigned to the edges incident to $v_{i}$ are $(0, a)$ and $(r-b, 0)$, where $a$ and $b$ are the weights of these edges. This yields that $c$ is a proper circular $r$-coloring at $v_{i}$ and that the compactness constraint is fulfilled at $v_{i}$. In the same way we check that the restrictions of compact circular coloring are satisfied by $c$ at vertices $v_{0}$ and $v_{1}$.

Note that if we follow the steps of the proof of Theorem 6 then we obtain an algorithm for compact circular coloring of odd cycles which runs in linear time. Moreover, since no circular $r$-coloring exists for any $r<\Delta(G, w)$, such an algorithm is 1.5 -approximate.

Corollary 7 There exists a 1.5-approximation algorithm running in $O(n)$ time for compact circular coloring of weighted odd cycles.

Now we prove that the problem of determining an optimal compact circular coloring of an odd cycle is NP-hard.

Theorem 8 The problem of determining if for a given weighted odd cycle $\left(C_{2 k+1}, w\right)$ there exists a compact circular $\Delta\left(C_{2 k+1}, w\right)$-coloring is NP-complete.

Proof: The proof that the considered problem belongs to NP is straightforward. We construct a reduction from the problem of determining whether a given weighted odd cycle admits a standard compact coloring, which is known to be NP-hard [3]. Consider a weighted cycle $(C, w)-$ an instance of this problem. Let $e_{1}, \ldots, e_{2 k+1}$ be the consecutive edges of the cycle and let $a_{i}$ be the weight of $e_{i}, i=1, \ldots, 2 k+1$. We construct an odd cycle $\bar{C}$ with $2 k+3$ edges $f_{1}, \ldots, f_{2 k+3}$ and weights $\bar{w}\left(f_{i}\right)=a_{i}$ for $i=1, \ldots, 2 k+1$ and $\bar{w}\left(f_{2 k+2}\right)=\bar{w}\left(f_{2 k+3}\right)=S$, where $S=a_{1}+\ldots+a_{2 k+1}$. Clearly, $\Delta(\bar{C}, \bar{w})=2 S$. We claim 
that $(C, w)$ admits any compact standard $r$-coloring if and only if $(\bar{C}, \bar{w})$ admits a compact circular $2 S$ coloring.

$(\Leftarrow)$ : Suppose that $c$ is a compact circular $2 S$-coloring of $(\bar{C}, \bar{w})$. We can assume, rotating the colors if needed, that $c\left(f_{2 k+2}\right)=(0, S)$ and $c\left(f_{2 k+3}\right)=(S, 2 S)$. Then, edge $f_{1}$ must obtain one of the colors $\left(0, a_{1}\right)$ or $\left(S-a_{1}, S\right)$ and edge $f_{2 k+1}$ must obtain one of the colors $\left(2 S-a_{2 k+1}, 2 S\right)$ or $\left(S, S+a_{2 k+1}\right)$. The compactness of $c$ gives us that

$$
\overline{\left(c\left(f_{1}\right) \cup \ldots \cup c\left(f_{2 k+1}\right)\right)} \subset C^{2 S}
$$

is an arc of length at most $S$. This yields that either $c\left(f_{1}\right)=\left(0, a_{1}\right)$ and $c\left(f_{2 k+1}\right)=\left(2 S-a_{2 k+1}, 2 S\right)$ or $c\left(f_{1}\right)=\left(S-a_{1}, S\right)$ and $c\left(f_{2 k+1}\right)=\left(S, S+a_{2 k+1}\right)$. Observe that the former case can be transformed to the latter by reversing and rotating the coloring, therefore we assume that $c\left(f_{1}\right)=\left(S-a_{1}, S\right)$ and $c\left(f_{2 k+1}\right)=\left(S, S+a_{2 k+1}\right)$. Using the compactness of $c$ again we obtain that $c\left(f_{i}\right)$ is a subinterval of $\left(a_{2 k+1}, 2 S-a_{1}\right)$ for every $i=1, \ldots, 2 k+1$. Therefore, we can assign color $c\left(f_{i}\right)$ to $e_{i}$ for $i=$ $1, \ldots, 2 k+1$ obtaining a compact standard $2 S$-coloring of $(C, w)$.

$(\Rightarrow)$ : Notice that if there exists any compact $r$-coloring of $(C, w)$, then there exists a compact $S$ coloring. Moreover, such a coloring can be transformed by shifting and if necessary reversing the colors to a compact $2 S$-coloring $c$, such that $c\left(e_{1}\right)=\left(S-a_{1}, S\right)$ and $c\left(e_{2 k+1}\right)=\left(S, S+a_{2 k+1}\right)$. We define an assignment $\bar{c}: E(\bar{C}) \mapsto \mathcal{A}\left(C^{2 S}\right)$ as follows: $\bar{c}\left(f_{i}\right)=c\left(e_{i}\right)$ for $i=1, \ldots, 2 k+1$ and $c\left(e_{2 k+2}\right)=(0, S)$, $c\left(e_{2 k+3}\right)=(S, 2 S)$. This gives us a compact circular $2 S$-coloring of $(\bar{C}, \bar{w})$.

We have proved that an optimal compact circular edge-coloring cannot be constructed in polynomial time, unless $\mathrm{P}=\mathrm{NP}$. However, we can construct a pseudo-polynomial algorithm that solves this problem.

Consider an odd cycle $(C, w)$ and denote its edges as $e_{1}, \ldots, e_{n}$. Let $(P, w)$ be a path of length $n$, with edges $f_{1}, \ldots, f_{n}$ and weights $w\left(f_{i}\right)=w\left(e_{i}\right)$. To construct an optimal compact circular edge-coloring of $(C, w)$ we first find for each $f_{i}$ the set of colors that can be assigned to that edge by some standard compact coloring $c$ of $(P, w)$ such that $c\left(f_{1}\right)=\left(0, w\left(f_{1}\right)\right)$ and $c\left(f_{j}\right)$ is an interval of $\mathbb{R}$ for all $j=1, \ldots, n$. For each $i=1, \ldots, n$ we construct a set $L[i]$ of the values that can be attained by the left endpoint of $c\left(f_{i}\right)$ in such a coloring. The sets $L[i]$ can be determined using the following dynamic programming formulas

$$
\begin{aligned}
L[1] & =\{0\}, \\
L[i+1] & =\left\{\ell-w\left(f_{i+1}\right): \ell \in L[i]\right\} \cup\left\{\ell+w\left(f_{i}\right): \ell \in L[i]\right\}, \quad i=1, \ldots, n-1 .
\end{aligned}
$$

The following proposition provides a relation between standard compact colorings of $(P, w)$ and compact circular colorings of $(C, w)$.

Proposition 9 Let $r \geq \Delta(C, w)$. A compact circular $r$-coloring of $(C, w)$ exists if and only if there exists a compact standard coloring $c$ of $(P, w)$ such that $c\left(f_{1}\right)=\left(0, w\left(f_{1}\right)\right)$ and $c\left(f_{n}\right)=\left(\right.$ ar $-w\left(f_{n}\right)$, ar $)$ for some integer $a$.

Proof: $(\Rightarrow)$ Consider a compact circular $r$-coloring $\bar{c}$ of $(C, w)$. Without loss of generality we can assume that $\bar{c}\left(e_{1}\right)=\left(0, w\left(e_{1}\right)\right)$ and $\bar{c}\left(e_{n}\right)=\left(r-w\left(e_{n}\right), r\right)$. We construct a compact coloring $c$ of $(P, w)$. First we put $c\left(f_{1}\right)=\left(0, w\left(f_{1}\right)\right)$ and then we define $c\left(f_{i+1}\right)$ using $c\left(f_{i}\right)$ for every $i=1, \ldots, n-1$. For every $i$ the left endpoint of $\bar{c}\left(e_{i+1}\right)$ is equal to the right endpoint of $\bar{c}\left(e_{i}\right)$, or the right endpoint of $\bar{c}\left(e_{i+1}\right)$ is equal to the left endpoint of $\bar{c}\left(e_{i}\right)$. In the first case we put $c\left(f_{i+1}\right)=\left(r_{i}, r_{i}+w\left(e_{i+1}\right)\right)$ and in the second case 
we put $c\left(f_{i+1}\right)=\left(l_{i}-w\left(e_{i+1}\right), l_{i}\right)$, where $\left(l_{i}, r_{i}\right)=c\left(f_{i}\right)$. It is easy to observe that $c$ defined in this way is a standard compact coloring of $(P, w)$ and that

$$
\bar{c}\left(e_{i}\right)=\left(\left[l_{i}\right]_{r},\left[r_{i}\right]_{r}\right) .
$$

Therefore, since $\bar{c}\left(e_{n}\right)=\left(r-w\left(e_{n}\right), r\right)$, we have $r_{n} \equiv 0(\bmod r)$, which implies $c\left(f_{n}\right)=($ ar $\left.w\left(f_{n}\right), a r\right)$ for some integer $a$.

$(\Leftarrow)$ Consider a compact coloring $c$ of $(P, w)$ such that

$$
c\left(f_{1}\right)=\left(0, w\left(e_{1}\right)\right), \quad c\left(f_{n}\right)=\left(a r-w\left(f_{n}\right), a r\right),
$$

where $a \in \mathbb{Z}$ and $r \geq \Delta(C, w)$. Denote $c\left(f_{i}\right)=\left(l_{i}, r_{i}\right)$ and put $\bar{c}\left(e_{i}\right)=\left(\left[l_{i}\right]_{r},\left[r_{i}\right]_{r}\right)$. We claim that $\bar{c}$ is a compact circular $r$-coloring of $(C, w)$. Using (3) we obtain $\bar{c}\left(e_{1}\right)=\left(0, w\left(e_{1}\right)\right)$ and $\bar{c}\left(e_{n}\right)=$ $\left(r-w\left(e_{n}\right), r\right)$. Therefore, the colors of $e_{1}$ and $e_{n}$ are not conflicting and the compactness criterion is satisfied in the common vertex of $e_{1}$ and $e_{n}$. Moreover, since $c$ is a compact coloring of path $P$ and $r \geq \Delta(C, w)$ coloring $\bar{c}$ must be proper and compact at every other vertex of the cycle $(C, w)$.

$\square$ Proposition 9 implies that once we have computed $L[n]$ we can find an optimal compact circular coloring by finding the minimum $r \geq \Delta(C, w)$ such that $a r-w\left(e_{n}\right) \in L[n]$ for some integer $a$.

Theorem 10 An optimal compact circular coloring of an odd cycle $(C, w)$ can be constructed in $O(n W)$ time, where $W=\sum_{i=1}^{n} w\left(e_{i}\right)$.

Proof: First, we derive the sets $L[i]$ for $i=1, \ldots, n$ using formulas (1) and (2). This can be completed in $O(n W)$ time, since $L[i]$ contains at most $2 W$ integers for every $i=1, \ldots, n$.

Then, we must find the minimum $r$, such that $r \geq \Delta(G, w)$ and $a r-w\left(e_{n}\right) \in L[n]$ for some integer $a$. If $\left(-w\left(e_{n}\right)\right) \in L[n]$ then obviously $r=\Delta(C, w)$. Otherwise, for each $\ell \in L[n]$ we derive

$$
r(\ell)=\left|\ell+w\left(e_{n}\right)\right| /\left\lfloor\left|\ell+w\left(e_{n}\right)\right| / \Delta(G, w)\right\rfloor,
$$

where $r(\ell)=+\infty$ if $\left|\ell+w\left(e_{n}\right)\right|<\Delta(G, w)$. It is easy to observe that $r(\ell)$ is the minimum real not less than $\Delta(G, w)$ such that $a \cdot r(\ell)=\ell+w\left(e_{n}\right)$ for some $a \in \mathbb{Z}$. Therefore, the optimal value $r$ is equal to

$$
r=\min \{r(\ell): \ell \in L(n)\}
$$

Notice that $\sum_{i=0}^{n-1} w\left(e_{i}\right) \in L(n)$ and $\sum_{i=0}^{n} w\left(e_{i}\right) \geq \Delta(G, w)$, which implies that $r$ defined by (4) satisfies $r<+\infty$. After we have computed $r$ we can find a compact circular $r$-coloring of $(C, w)$. First, backtracking the algorithm for computing $L[i]$, we find a standard coloring $c$ of $(P, w)$ such that $c\left(f_{1}\right)=\left(0, w\left(f_{1}\right)\right)$ and $c\left(f_{n}\right)=\left(a r-w\left(f_{n}\right), a r\right)$ for some integer $a$. Finally, putting $\bar{c}\left(e_{i}\right)=\left(\left[l_{i}\right]_{r},\left[r_{i}\right]_{r}\right)$, where $\left(l_{i}, r_{i}\right)=c\left(f_{i}\right)$ for $i=1, \ldots, n$, we obtain a compact circular $r$-coloring $\bar{c}$, in the same way as in the proof of Proposition 9 .

The results we have proved so far provide that odd cycles can be colored in a compact circular way in polynomial time, while the problem of determining whether for an input graph there exists any compact circular coloring is NP-complete in general. One may ask, how the structure of the cycle must be made more complicated to make this problem hard. The following theorem proves that it is NP-hard for an odd cycle with a path $P_{2}$ connected to a vertex of the cycle. 


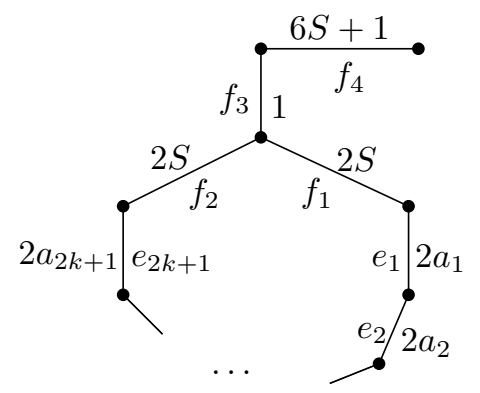

Fig. 2: A graph $(G, \bar{w})$ constructed in the proof of Theorem 11

Theorem 11 The following problem is NP-complete: "Given weighted graph $(G, w)$, where G has a structure of an odd cycle with a path $P_{2}$ connected to one of its vertices. Does there exist a real $r>0$ such that $(G, w)$ admits a compact circular $r$-coloring?".

Proof: In the same way as in the proof of Theorem 8 we reduce the problem of determining if there exists a compact $r$-coloring of $\left(C_{2 k+1}, w\right)$ for some $r$ to the considered problem. Let $a_{1}, \ldots, a_{2 k+1}$ be the weights of consecutive edges of $\left(C_{2 k+1}, w\right)$ and let $S=a_{1}+\ldots+a_{2 k+1}$. Consider a weighted graph $(G, \bar{w})$ depicted in Fig. 2. We claim that $(G, \bar{w})$ admits a compact circular $r$-coloring for some $r$ if and only if $\left(C_{2 k+1}, w\right)$ is standard compact $q$-colorable for some $q$.

$(\Rightarrow)$ : Suppose $c$ is a compact circular $r$-coloring of $(G, \bar{w})$. Clearly, $r \geq \Delta(G, \bar{w})=6 S+2$. Without loss of generality, rotating the coloring if needed, we may assume that either $c\left(f_{1}\right)=(0,2 S)$ and $c\left(f_{2}\right)=$ $(2 S, 4 S)$ or $c\left(f_{1}\right)=(0,2 S)$ and $c\left(f_{2}\right)=(2 S+1,4 S+1)$.

Consider the first case. The color of edge $e_{1}$ is equal to either $\left(r-2 a_{1}, r\right)$ or $\left(2 S, 2 S+2 a_{1}\right)$ and the color of $e_{2 k+1}$ is either $\left(2 S-2 a_{2 k+1}, 2 S\right)$ or $\left(4 S, 4 S+2 a_{2 k+1}\right)$. However, $\overline{\left(c\left(e_{1}\right) \cup \ldots \cup c\left(e_{2 k+1}\right)\right)}$ is an arc of length at most $2 S$, since $c$ is compact, therefore we get that $c\left(e_{1}\right)=\left(2 S, 2 S+2 a_{1}\right)$ and $c\left(e_{2 k+1}\right)=\left(2 S-2 a_{2 k+1}, 2 S\right)$. Moreover, $c\left(e_{i}\right) \subset(0,4 S)$ for $i=1, \ldots, 2 k+1$, which yields that $c$ can be transformed to a compact $4 S$-coloring of $\left(C_{2 k+1}, w\right)$.

We claim that the second case cannot occur. If we suppose that $c\left(f_{1}\right)=(0,2 S)$ and $c\left(f_{2}\right)=(2 S+$ $1,4 S+1)$, in the same way we obtain that $c\left(e_{1}\right)=\left(2 S, 2 S+2 a_{1}\right), c\left(e_{2 k+1}\right)=\left(2 S-2 a_{2 k+1}+1,2 S+1\right)$. However, compactness of $c$ yields that $c\left(e_{i}\right) \subset(0,4 S+1), i=1, \ldots, 2 k+1$ implying that the arcs $c\left(e_{i}\right)$, $i=1, \ldots, 2 k+1$ either all begin in even points or all begin in odd points - a contradiction.

$(\Leftarrow)$ : Suppose that there exists a compact standard $r$-coloring of $\left(C_{2 k+1}, w\right)$. Then we can transform it, in the same way as in the proof of Theorem 8 , to a compact circular $(6 S+2)$-coloring.

\section{Concluding Remarks}

We have studied the complexity of the problem of circular edge-coloring of weighted graphs. The former results of [11] give a polynomial-time algorithm for circular coloring of outerplanar bipartite graphs, while coloring general bipartite graphs was proven to be NP-hard [9, 12]. The theorems of the last section extend these results and yield a nearly complete classification of the complexity of the problem of coloring nonbipartite graphs. We have proved that it is NP-hard to optimally color weighted odd cycles. Moreover, Theorem 11 implies that it is not possible to provide a polynomial-time algorithm that constructs any 
compact circular coloring of non-bipartite graphs from any significantly wider class than odd cycles. This also justifies, why in Theorem 6 and Corollary 7 we have considered such simple graphs as odd cycles.

\section{References}

[1] Asratian, A. S., Denley, T. R. J., Häggkvist, R.: Bipartite Graphs and Their Applications, Cambridge Univ. Press (1998).

[2] Giaro, K.: NP-hardness of compact scheduling in simplified open and flow shops European J. Oper. Res. 130 (2001) 90-98.

[3] Giaro, K., Task Scheduling Without 2-Sided Waiting Periods on Dedicated Processors (in Polish). Ph. D. Thesis, Gdańsk Univ. of Tech. Gdańsk (1999).

[4] Giaro, K., Interval edge-coloring of graphs, in: Graph Colorings, (M. Kubale, Ed.), Contemporary Mathematics 352, AMS (2004), 105-121.

[5] Hackmann, A., Kemnitz, A., The circular chromatic index, Discrete Math. 286 (2004) 89-93.

[6] Jensen, T.R., Toft, B., Graph Coloring Problems, Wiley (1994).

[7] Nadolski, A., Compact cyclic edge-coloring of graphs, Discrete Math. 3082008 2407-2417.

[8] Nadolski, A., Chromatic Scheduling in Cyclic Manufacturing Systems (in Polish), Ph. D. Thesis, Gdańsk Univ. of Tech. Gdańsk (2005).

[9] Kubale, M., Nadolski, A., Chromatic scheduling in a cyclic open shop, European J. Oper. Res. 164 (2005) 585-591.

[10] Sevastyanov, S. V., Interval colorability of the edges of a bipartite graph (in Russian), Metody Diskret. Analiz. 50 (1990) 61-72.

[11] Solot, Ph., de Werra, D., Compact cylindrical scheduling, SIAM J. Discr. Math. 4 (1991) 528-534.

[12] Solot, Ph., de Werra, D., Some graph-theoretical models for scheduling in automated production systems, Networks 23 (1993) 651-660. 